\title{
TOWARDS THE INTEGRATION OF SAR TOMOGRAPHY AND PSI FOR IMPROVED DEFORMATION ASSESSMENT IN URBAN AREAS
}

\author{
Muhammad Adnan Siddique $^{(1)}$, Irena Hajnsek ${ }^{(1,3)}$, Urs Wegmüller ${ }^{(2)}$, and Othmar Frey ${ }^{(1,2)}$ \\ ${ }^{(1)}$ Earth Observation \& Remote Sensing, ETH Zurich, Switzerland, Email: \{siddique,hajnsek,frey\}@ifu.baug.ethz.ch \\ ${ }^{(2)}$ Gamma Remote Sensing AG, Gümligen, Switzerland, Email: \{wegmuller,frey\}@ gamma-rs.ch \\ ${ }^{(3)}$ German Aersospace Center - DLR, Oberpfaffenhofen, Germany
}

\begin{abstract}
Persistent scatterer interferometry (PSI) typically rejects the range-azimuth pixels containing multiple scatterers, such as in a layover scenario. Since layovers occur frequently in urban areas, a significant number of candidates may get rejected. SAR tomography allows for resolving layover and has thus the potential to extend the spatial sampling of deformation measurements to layoveraffected areas. Using extended phase models, also taking into account temperature, an improved simultaneous estimation of elevation, deformation velocity, and temperature-induced scatterer displacement is possible. This paper explores the combined use of PSI and SAR tomography for deformation analysis in urban areas, using a multibaseline and multitemporal interferometric stack of stripmap TerraSAR-X images acquired over the city of Barcelona.
\end{abstract}

Key words: SAR tomography; PSI; DIn-SAR; multibaseline interferometry.

\section{INTRODUCTION}

Persistent scatterer interferometry (PSI) [1, 2] is an established tool for radar-based deformation assessment in urban areas. Using an interferometric time-series of multibaseline SAR images, PSI allows retrieval of radar lineof-sight (LOS) deformation parameters. PSI-based analysis entails a preliminary identification of PS candidates based on methods such as spectral diversity and temporal variability of the backscattering [2]. PSI relies on the assumption of a single dominant scatterer in a rangeazimuth pixel. Therefore, the pixels with multiple scatterers (such as in a ground-to-facade layover) are typically rejected. SAR tomography $[3,4,5]$ has the potential to overcome this limitation. It has the ability to retrieve the elevation and deformation parameters for multiple scatterers in the same resolution cell, as shown in different contributions such as $[6,7,8,9]$.

In continuation of our earlier work $[10,11,12]$ towards the integration of SAR tomography into the operational 'Interferometric Point Target Analysis (IPTA)' [2] tool for PSI processing, this paper presents our current investigations. We report results on an interferometric stack of X-band stripmap SLCs acquired over an urban area. A PSI solution is first obtained using the IPTA framework. SAR tomographic inversion is applied next independently of the PSI solution, though keeping the same reference layer and reference point. We implement tomography with three different phase models, and compare the simultaneously estimated deformation parameters with those obtained iteratively with the PSI solution. In this paper, we focus on a particular high-rise building where potentially deformation and thermal expansion are to be expected.

\section{SAR TOMOGRAPHIC MODEL}

Considering a stable point target source in a given rangeazimuth resolution cell, the mathematical model for conventional SAR tomography (3D SAR imaging) can be written as $[5,13]$ :

$$
y_{n}=\int_{\triangle s} \gamma(s) \exp \left[-j \varphi_{n}(s)\right] \mathrm{d} s
$$

where $y_{n}$ is the $n^{\text {th }}$ single-look-complex (SLC) value from a coregistered stack containing $N$ images, $n=$ $0,1, \ldots, N-1, \gamma(s)$ is the reflectivity profile along the elevation $s$, and $\varphi_{n}$ is the following interferometric phase term [5]:

$$
\begin{aligned}
\varphi_{n}(s) & =2 k\left(r_{n}(s)-r_{0}(s)\right) \\
& \approx 2 k\left[\frac{s^{2}}{2\left(r_{0}-b_{n}^{\|}\right)}-\frac{b_{n}^{\perp} s}{r_{0}-b_{n}^{\|}}\right]
\end{aligned}
$$

where $k$ is the central wavenumber, and $r_{n}$ is the range distance from sensor $n$ to scatterer at elevation $s$. In this context, elevation refers to the distance along the perpendicular to line-of-sight (PLOS) direction. The reference acquisition in the interferometric stack is represented with $n=0 . b_{n}^{\perp}$ and $b_{n}^{\|}$represent the orthogonal 

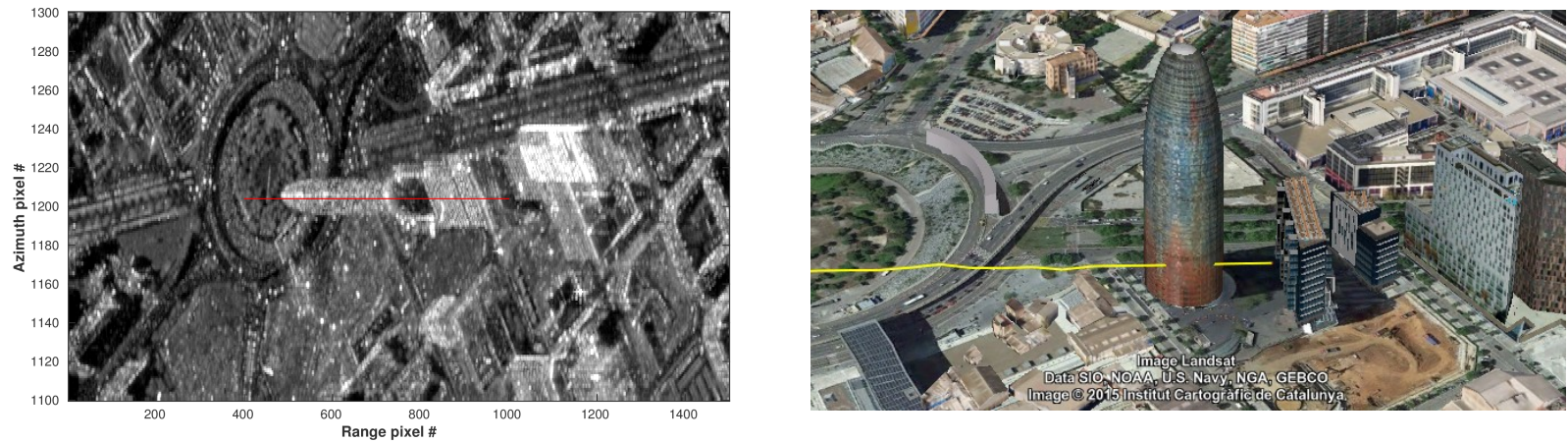

Figure 1. Left: SAR average backscatter image of the observed area. The high-rise building suffering from layover is the Torre Agbar tower. The red line marks the extent of an echo whose tomograhic inversion is presented afterwards. Right: Google Earth view of the corresponding neighborhood. The yellow line marks the approximate on-ground projection of the slant-range vector.

and parallel baseline components between the sensor $n$ and the reference image, respectively.

The interferometric phase in eq. (2) depends only on the sensor-to-target geometry, and assumes a stationary scatterer. The scatterer may, however, be subject to displacement over time, which would incur an additional phase variation. Assuming a linear deformation model over time, the extended phase model yields:

$$
\varphi_{n}(s, \nu)=2 k\left[\Delta r_{n}(s)+\nu t_{n}\right]
$$

where $\nu$ is then the average deformation velocity. The SLC value of the $n^{t h}$ sensor can now be written as:

$$
y_{n}=\iint_{\triangle s, \triangle \nu} \gamma(s, \nu) \exp \left[-j \varphi_{n}(s, \nu)\right] \mathrm{d} s \mathrm{~d} \nu
$$

This tomographic model is generally known as differential tomography $[14,15,16]$.

As reported in different PSI studies (such as [17][18]), scatterers may also be subject to an additional phase variation due to thermal expansion of buildings. In this case, the model can be further extended to:

$$
\varphi_{n}(s, \nu, \kappa)=2 k\left[\triangle r_{n}(s)+\nu t_{n}+\frac{1}{2 k} \kappa \tau_{n}\right]
$$

where $\tau_{n}$ is the local temperature corresponding to the time of the $n^{t h}$ image acquisition, and $\kappa$ represents the phase-to-temperature sensitivity. A more general tomographic model can now be concisely written as:

$$
y_{n}=\int_{\triangle \mathbf{p}} \gamma(\mathbf{p}) \exp \left[-j \varphi_{n}(\mathbf{p})\right] \mathrm{d} \mathbf{p}
$$

where $\gamma(\mathbf{p})$ and $\mathbf{p}=[s, \nu, \kappa]$ are the unknown scatterer reflectivity and parameter vector, respectively. Various inversion methods have been proposed (such as [19, 12, 4, 3]) for estimating $\gamma(\mathbf{p})$ and $\mathbf{p}$. In this work, we perform single-look conventional beamforming (which is used as the objective function for the maximisation applied for the retrieval of the unknown parameters). The estimated reflectivity is given by,

$$
\hat{\gamma}(\mathbf{p})=\mathbf{a}^{H}(\mathbf{p}) \mathbf{y}
$$

where $\mathbf{y}$ is the column vector containing the SLC values, and $\mathbf{a}$ is the steering vector:

$$
\mathbf{a}(\mathbf{p})=\left[\begin{array}{llll}
1 & e^{-j \varphi_{1}(\mathbf{p})} & \ldots & e^{-j \varphi_{N-1}(\mathbf{p})}
\end{array}\right]^{\mathrm{T}}
$$

The unknown parameter vector is estimated using the following maximization:

$$
\hat{\mathbf{p}}=\underset{\mathbf{p}}{\operatorname{argmax}}\left(\frac{\hat{\gamma}(\mathbf{p})}{\|\mathbf{a}(\mathbf{p})\|\|\mathbf{y}\|}\right)
$$

In practice, the SLC vector $\mathbf{y}$ generally suffers from noise, especially in areas of low-backscatter or shadow regions. The noise component may lead to poor tomographic inversion. A given resolution cell may not even contain a temporally coherent scatterer. Ignoring any phase miscalibration, we formulate the detection problem as a decision between two hypotheses, $H_{0}$ and $H_{1}$.

$$
\begin{aligned}
& H_{0}: \mathbf{y}=\mathbf{w} \\
& H_{1}: \mathbf{y}=\gamma\left(\mathbf{p}_{1}\right) \mathbf{a}\left(\mathbf{p}_{\mathbf{1}}\right)+\mathbf{w}
\end{aligned}
$$

where $\mathbf{w}$ represents the noise term, generally modelled as an additive $N$-dimensional circular Gaussian random vector. Using a threshold $T$, we use the Generalized Likehood Ratio Test (GLRT) [20][6] for this detection problem, as follows:

$$
\underset{\mathbf{p}}{\operatorname{argmax}}\left(\frac{\hat{\gamma}(\mathbf{p})}{\|\mathbf{a}(\mathbf{p})\|\|\mathbf{y}\|}\right) \underset{H_{1}}{\stackrel{H_{0}}{\lessgtr}} T
$$

It can be noted here that equations 9 and 11 require maximisation of the same objective function. Therefore, only a single maximisation needs to be carried out for each resolution cell. A scatterer is considered 'detected' only when the hypothesis $H_{1}$ is accepted, i.e. when the maximum value of the objective function exceeds the threshold. The elevation and deformation parameters for the 

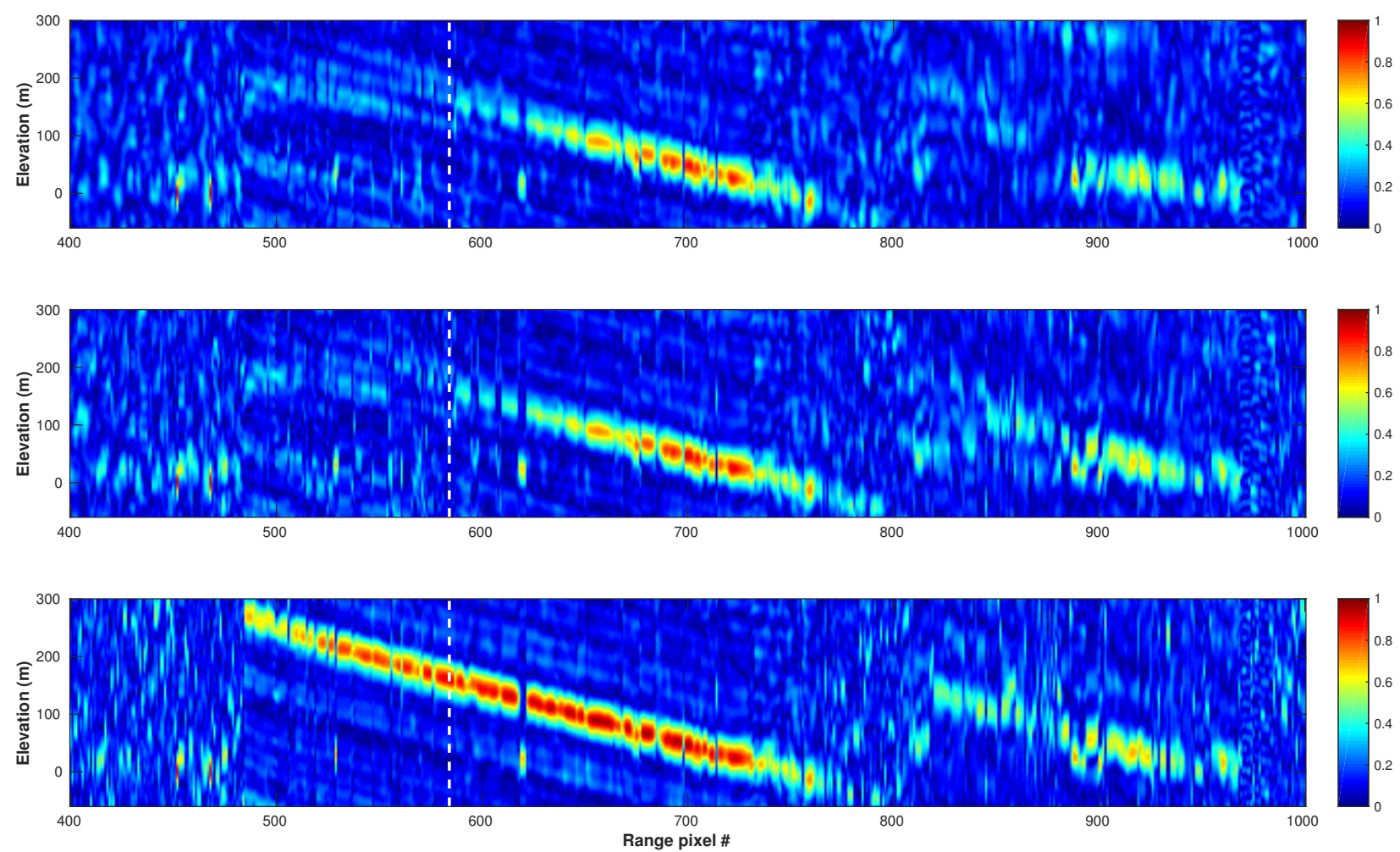

Figure 2. Tomograms obtained with the maximization of the objective function based on tomographic inversion with beamforming (see eq. 9). The elevation axis is perpendicular to line-of-sight (PLOS). Top: Only the sensor-to-target geometry dependent phase is modelled (P1). Middle: The phase model additionally provides for a linear deformation term (P2). Bottom: The phase model is further extended to include a thermal expansion induced phase term (P3). The color scale represents the reflectivity: $\hat{\gamma}(\mathbf{p}) /\|\mathbf{a}(\mathbf{p})\|\|\mathbf{y}\|$.

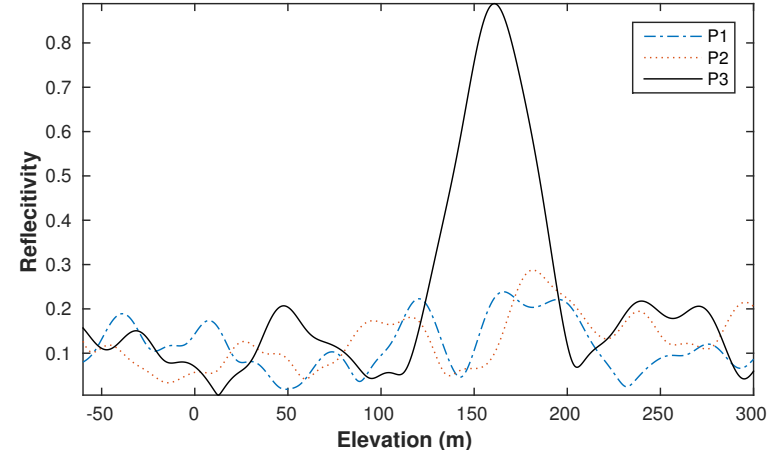

Figure 3. The estimated elevation profiles, corresponding to each phase model (P1, P2, and P3), for the transect shown in white dashed line in Fig. 2.

detected scatterer (DS) are simultaneously estimated (as the elements of the parameter vector corresponding to the maximum of the objective function).

The detection threshold impacts the probabilities of detection and false alarm. It is not within the scope of this paper to discuss the choice of the threshold; interested readers are referred to [20] for further details and a comparison with other detection schemes.

\section{PHASE CALIBRATION}

The interferometric data stack needs to be precisely phase calibrated. Atmospheric phase screen (APS) has to be estimated and compensated for each layer in the stack prior to tomographic inversion. An accurate estimation of the APS is restricted by the presence of various sources of phase noise, such as temporal and geometric decorrelation, orbital errors, etc. This preprocessing step entails a preliminary PSI analysis. In our work, we use the Interferometric Point Target Analysis (IPTA) [2] to obtain an initial PSI solution. A set of PS candidates is selected based on their low spectral diversity and high temporal stability of the backscatter. The orbital and topographic phases are simulated and subtracted from the complexvalued point interferograms. SRTM DEM is used for the simulation of topography, as an estimate of the initial heights (although it is not necessary in principle). The point differential interferograms are analysed next. The atmospheric phase exhibits low-frequency behaviour in the spatial domain, but high-frequency in temporal domain (as the acquisitions are repeat-pass). Once an acceptable PSI solution is obtained, the low-frequency spatial behaviour of the atmospheric phase allows extrapolation of the point-wise estimations to the surrounding non-PS as well. Further details on the IPTA framework 

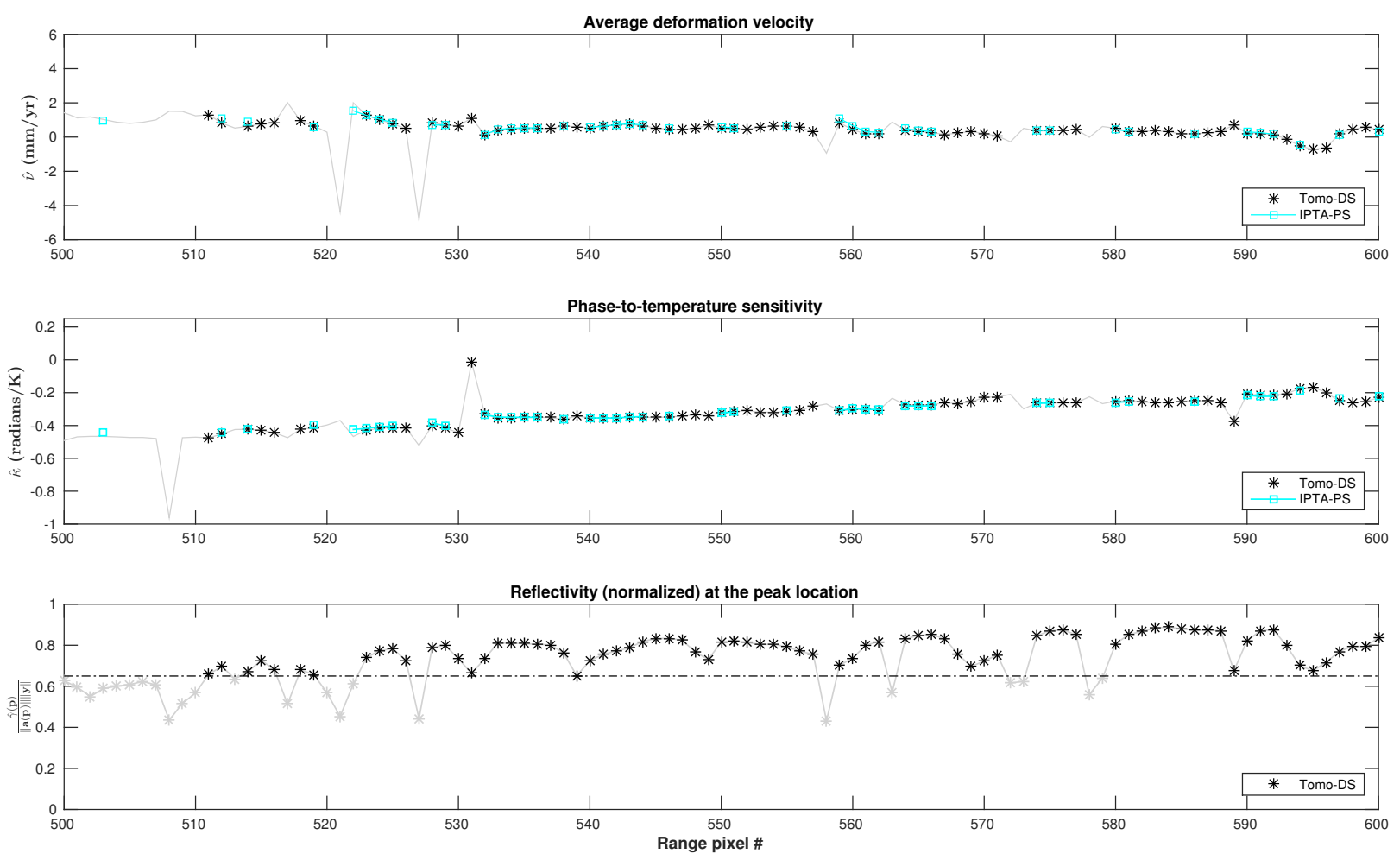

Figure 4. A comparison between the deformation parameters estimated using two different approaches: 1) an iterative PSI approach (IPTA), and 2) SAR tomography. In both cases, we have used the extended phase model (P3) i.e. modelling scatterer elevation, deformation and thermal expansion induced phase variation. PSI estimates are obtained iteratively using the IPTA framework. For the case of tomographic inversions, the estimates are obtained simultaneously with the maximisation of the beamforming function (eq. 9). Top: The estimated deformation velocity. Middle: The estimated phase-to-temperature sensitivity. Bottom: The reflectivity at the peak location. A threshold is set at 0.65 for the detection of a scatterer in each resolution cell.

are referred to $[2,21,22]$.

\section{DATA STACK}

The interferometric data stack used in this work comprises of 50 repeat-pass TerraSAR-X stripmap acquisitions. The images were acquired over the city of Barcelona over a time span of approximately 5 years. The total orthogonal baseline is $503.2 \mathrm{~m}$. The distribution of both the temporal and spatial baselines are highly nonuniform.

\section{RESULTS}

Fig. 1 shows the Torre Agbar tower; the layover cast by the tower is clearly visible in the SAR image. The tomograms in Fig. 2 show the focusing performance obtained for the three phase models (P1: elevation only, P2: elevation and linear deformation, P3: elevation, linear deformation, and thermal expansion). The elevation profiles for the transect marked in white dashed line in the tomograms are shown in Fig. 3. We obtained estimates of the elevation, deformation velocity and thermal expansion using the IPTA framework as well. A comparison of the estimates of the deformation parameters obtained with IPTA for the PSs, and those obtained with tomographic inversion on the DSs, is given in Fig. 4.

\section{DISCUSSION}

We performed inversions for each of the three tomographic models presented in section 2: P1: elevation only $(\mathbf{p}=[s]), \mathbf{P} 2$ : elevation and linear deformation $(\mathbf{p}=[s, \nu])$, P3: elevation, linear deformation, and thermal expansion $(\mathbf{p}=[s, \nu, \kappa])$. Fig. 2 shows the tomograms obtained individually with each model. The top and middle tomograms, corresponding to P1 and P2 respectively, clearly show that the upper part of the building is not properly reconstructed. It implies that these two models (which consider only the sensor-to-target geometry and linear deformation induced phase changes) are not well-suited for this building. The tomogram corresponding to $\mathrm{P} 3$, which additionally models a thermal ex- 
pansion induced phase change, shows a marked improvement. The facade of the building, from the top to bottom, has been nicely focused in elevation. We closely observe the transect marked in white dashed line, and compare the elevation profiles for each model in Fig. 3. A wellfocused elevation profile is estimated only for P3.

With tomograhic inversion, the unknown parameter vector is estimated at the peak of $\hat{\gamma}(\mathbf{p})$. We use the GLRT (eq. 11) for the detection of the scatterers. The detection threshold can be empirically selected between 0 and 1. We set the threshold at $T=0.65$. A comparison among the deformation parameters' estimates obtained with IPTA on the selected PSs, and with tomographic inversion on DSs, is presented in Fig. 4. The estimates are mostly consistent. The PSI solution obtained with the IPTA framework involves an 'iterative' regression analysis for the thermal expansion term and the combined elevation and deformation term, respectively; tomographic inversion, on the other hand, provides a 'simultaneous' estimation. Moreover, there is a higher density of DSs than PSs, i.e. higher deformation sampling. There are a few resolution cells getting detected as PS but not as DS, which raises a point of inquiry whether the threshold for the detection should be relaxed.

\section{CONCLUSION}

This paper provides an analysis of three different phase models for tomographic inversion of an interferometric stack of TerraSAR-X data acquired over Barcelona. The results show that in case of high-rise buildings, it may be necessary to model thermal expansion induced phase changes. Insufficient phase modelling leads to improper focusing and hence missed detection. We have compared the velocity and elevation parameters estimated from both a tomographic model and an iteratively obtained PSI solution using an extended phase model. The results are consistent. A detailed assessment of the detection of double-scatterers using a more appropriate layover scenario, and simultaneous retrieval of their individual deformation parameters, will be part of our future work.

\section{ACKNOWLEDGMENTS}

This research project has been funded by the Swiss Space Office, State Secretariat for Education and Research of the Swiss Confederation (SER/SSO), under the MdP2012 initiative. TerraSAR-X SAR data used in this project was obtained courtesy of the German Aerospace Center DLR under proposal MTH1717. SRTM is copyright USGS.

\section{REFERENCES}

[1] A. Ferretti, C. Prati, and F. Rocca. Permanent scatterers in SAR interferometry. IEEE Trans. on Geosc. and Remote Sens., 39(1):8-20, 2001.
[2] C. Werner, U. Wegmüller, T. Strozzi, and A. Wiesmann. Interferometric point target analysis for deformation mapping. In Proc. IEEE Int. Geosci. Remote Sens. Symp., pages 4362-4364.

[3] F. Lombardini, F. Cai, and D. Pasculli. Spaceborne 3-D SAR tomography for analyzing garbled urban scenarios: Single-look superresolution advances and experiments. IEEE Jour. of Sel. Topics in Appl. Earth Observ. and Remote Sens., 6(2):960 968, 2013.

[4] X. Zhu and R. Bamler. Very high resolution spaceborne SAR tomography in urban environment. IEEE Trans. on Geosc. and Remote Sens., 48(12):4296-4308, Dec 2010.

[5] G. Fornaro, F. Serafino, and F. Soldovieri. Three-dimensional focusing with multipass SAR data. IEEE Trans. on Geosc. and Remote Sens., 41(3):507-517, March 2003.

[6] A. Pauciullo, D. Reale, A. De Maio, and G. Fornaro. Detection of double scatterers in SAR tomography. IEEE Trans. on Geosc. and Remote Sens., 50(9):3567-3586, Sept 2012.

[7] X. Zhu and R. Bamler. Super-resolution power and robustness of compressive sensing for spectral estimation with application to spaceborne tomographic SAR. IEEE Trans. on Geosc. and Remote Sens., 50(1):247-258, Jan 2012.

[8] G. Fornaro, D. Reale, and S. Verde. Bridge thermal dilation monitoring with millimeter sensitivity via multidimensional SAR imaging. IEEE Geosci. and Remote Sens. Lett., 10(4):677-681, 2013.

[9] F. Lombardini and M. Pardini. Superresolution differential tomography: Experiments on identification of multiple scatterers in spaceborne SAR data. IEEE Trans. on Geosc. and Remote Sens., 50(4):1117-1129, April 2012.

[10] O. Frey and E. Meier. 3D SAR imaging of a forest using airborne MB-SAR data at L-and P-band: Data processing and analysis. In 8th Euro. Conf. on SAR (EUSAR), pages 1-4. VDE, 2010.

[11] O. Frey, I. Hajnsek, U. Wegmüller, and C. Werner. SAR tomography based 3-D point cloud extraction of point-like scatterers in urban areas. In Proc. IEEE Int. Geosci. Remote Sens. Symp., pages 1313-1316, 2014.

[12] O. Frey, M. Siddique, I. Hajnsek, U. Wegmüller, and C. Werner. Combining SAR tomography and a PSI approach for high-resolution 3-D imaging of an urban area. In Proc. 10th European Conf. on SAR, pages 1045-1048, 2014.

[13] G. Fornaro and F. Serafino. Imaging of single and double scatterers in urban areas via SAR tomography. IEEE Trans. on Geosc. and Remote Sens., 44(12):3497-3505, 2006.

[14] F. Lombardini. Differential tomography: A new framework for SAR interferometry. IEEE Trans. on Geosc. and Remote Sens., 43(1):37-44, 2005. 
[15] G. Fornaro, F. Lombardini, A. Pauciullo, D. Reale, and F. Viviani. Tomographic processing of interferometric SAR data: Developments, applications, and future research perspectives. IEEE Signal Process. Mag., 31(4):41-50, July 2014.

[16] X. Zhu and R. Bamler. Compressive sensing for high resolution differential SAR tomography - the SL1MMER algorithm. In Proc. IEEE Int. Geosci. Remote Sens. Symp., pages 17-20, July 2010.

[17] O. Monserrat, M. Crosetto, M. Cuevas, and B. Crippa. The thermal expansion component of persistent scatterer interferometry observations. IEEE Trans. on Geosc. and Remote Sens., 8(5):864868, September 2011.

[18] M. Crosetto, O. Monserrat, M. Cuevas-González, N. Devanthéry, G. Luzi, and B. Crippa. Measuring thermal expansion using X-band persistent scatterer interferometry. ISPRS Jour. of Photogrammetry and Remote Sens., 100:84 - 91, 2015.

[19] O. Frey, I. Hajnsek, and U. Wegmüller. Spaceborne SAR tomography in urban areas. In Proc. IEEE Int. Geosci. Remote Sens. Symp., pages 69-72, July 2013.

[20] A. De Maio, G. Fornaro, and A. Pauciullo. Detection of single scatterers in multidimensional SAR imaging. IEEE Trans. on Geosc. and Remote Sens., 47(7):2284-2297, July 2009.

[21] U. Wegmuller, D. Walter, V. Spreckels, and C.L. Werner. Nonuniform ground motion monitoring with TerraSAR-X persistent scatterer interferometry. IEEE Trans. on Geosc. and Remote Sens., 48(2):895-904, Feb 2010.

[22] O. Frey, U. Wegmuller, and C. Werner. Terrain motion measurements over European urban areas using persistent scatterer interferometry. In Proc. IEEE Int. Geosci. Remote Sens. Symp., pages 7565-7568, July 2012. 